\title{
Continuation of Bifurcations of Periodic Orbits for Large-Scale Systems*
}

\author{
M. Net ${ }^{\dagger}$ and J. Sánchez ${ }^{\dagger}$
}

\begin{abstract}
A methodology to track bifurcations of periodic orbits in large-scale dissipative systems depending on two parameters is presented. It is based on the application of iterative Newton-Krylov techniques to extended systems. To evaluate the action of the Jacobian it is necessary to integrate variational equations up to second order. It is shown that this is possible by integrating systems of dimension at most four times that of the original equations. In order to check the robustness of the method, the thermal convection of a mixture of two fluids in a rectangular domain has been used as a test problem. Several curves of codimension-one bifurcations, and the boundaries of an Arnold's tongue of rotation number $1 / 8$, have been computed.
\end{abstract}

Key words. continuation methods, numerical computation of invariant objects, periodic orbits, bifurcation tracking, extended systems, Newton-Krylov methods, variational equations

AMS subject classifications. 37G15, 37L15, 37L20, 37M05, 37M20, 65P30, 65Z05, 76D05, 76E06, 76M22

DOI. $10.1137 / 140981010$

1. Introduction. The study of dynamical systems involves, in addition to pure numerical simulations, the computation of invariant manifolds (fixed points, periodic orbits, invariant tori, etc.) and the connections among them (homo- and heteroclinic orbits and heteroclinic chains), the investigation of the stability of these objects, and the examination of their bifurcations when the parameters present in the system are varied. These essential tools help to understand the full dynamics of the system and its dependence on the parameters. The existence of robust continuation and bifurcation packages such as AUTO [10], CONTENT [22], MATCONT [7], etc., allows many of these calculations to be almost routinely performed for moderate-dimensional systems of ordinary differential equations (ODEs). These packages include the continuation of codimension-one bifurcations of fixed points, and even of periodic orbits, and some also include the detection and analysis of codimension-two points. They implement direct solvers for the linear systems involved in the computations, and find the full spectrum when solving eigenvalue problems to study of the stability of the invariant objects.

The theory of the extended systems used in these packages to follow bifurcations of fixed points is well developed and can be found in, among others, [42, 26, 18, 53, 29, 5, 16]. The bordered systems for periodic orbits, based on boundary value problems, are analyzed in [11]. In this latter case piecewise collocation in time is used instead of shooting methods.

The difficulties in the application of these methodologies to high-dimensional systems, obtained in most cases by discretizing systems of partial differential equations (PDEs), come

\footnotetext{
${ }^{*}$ Received by the editors August 4, 2014; accepted for publication (in revised form) by D. Barkley February 11, 2015; published electronically April 14, 2015. This work was supported by MCYT/FEDER projects MTM2010-16930 and FIS2013-40674-P and by Catalonia GENCAT under project 2014-SGR-1145.

http://www.siam.org/journals/siads/14-2/98101.html

${ }^{\dagger}$ Departament de Física Aplicada, Universitat Politècnica de Catalunya, Jordi Girona Salgado, 1-3, Campus Nord, Mòdul B-4, 08034 Barcelona, Spain (marta.net@upc.edu, juan.j.sanchez@upc.edu).
} 
from the implementation of the linear algebra. Relatively new techniques, based on Krylov or Arnoldi methods [30, 31], have allowed the study of large systems; in many cases in computational fluid dynamics. Inexact Newton-Krylov methods are used to find mainly fixed points, along with subspace iteration or Arnoldi methods to study their stability [12, 2, 24, 9]. Preconditioning is usually needed to overcome the difficulty of achieving fast convergence of these iterative methods when computing steady solutions.

The computation of periodic orbits in high-dimensional systems is more recent. NewtonPicard algorithms were used in [25] and implemented in the package PDECONT, a limited memory Broyden method was applied in [46], and Newton-Krylov techniques were used in [38]. Nontrivial extensions to parallel-shooting [36] and to the calculation of the coefficients of a normal form at a multicritical periodic orbit [41] were later implemented. The continuation of invariant tori was considered first in [40] and then improved with a parallel method in [37]. The computation of two-dimensional unstable manifolds of periodic orbits was tackled in [47].

The continuation of bifurcations of fixed points of large-scale dynamical systems has its starting point in the work of Cliffe [4]. He computed saddle-node and pitchfork bifurcations in the Taylor-Couette problem, a classical subject in fluid mechanics, using finite elements. He also detected the presence of cusp points. The only general purpose package designed for large-scale applications, LOCA [33], allows tracking steady solutions, their codimension-one points [34], and phase transitions. As far as we know, the only attempt to compute bifurcations points of periodic orbits was reported in [13]. The authors obtained period-doubling points of cycles by using Newton-Picard methods in an extension of their previous work on the computation of limit cycles [25].

Two recent reviews $[19,9]$ present the current status of the application of dynamical systems techniques in fluid dynamics, including several examples showing the relevance of these methods in the understanding of turbulence.

In this paper we develop a new efficient methodology for the continuation of the codimension-one bifurcations of periodic orbits, including the pitchfork bifurcation present in reflection-symmetric systems. It is based on the combination of Newton-Krylov techniques applied to extended systems and the integration of systems of variational equations up to second order. Shooting methods are used to solve the boundary value problems defining the periodic orbits. Collocation methods in time would add a new dimension to discretize, leading to huge systems to solve. The initial dimension of the system, already large, would be multiplied by the number of collocation points. The extended systems used here are adapted from those usually found in the literature for fixed points of maps [21]. A deflation term is needed in some cases to remove the trivial +1 multiplier of the periodic orbits. As far as we know, this is the first time these techniques are used in the numerical study of curves of bifurcations in PDEs. It will be shown that once the problem has been discretized, it is only necessary to integrate systems of ODEs of dimension at most four times that of the original system. This minimizes the computational cost.

Two main tools are required to implement the algorithms presented here: a generic continuation code and a time integrator for the particular problem at hand, including the required variational equations. Section 2 describes a general Newton-Krylov continuation code, without referring to any specific software package, and it is recalled how it can be applied to compute periodic orbits. With this information it is easy to see how to adapt such a code to 
follow loci of bifurcation points of cycles. It is also assumed that the time integrator properly solves initial value problems. If a multistep method is used for a system of truncated PDEs, then a mechanism for starting the time integration, for instance with variable step size and/or variable order, is essential.

The thermal convection of a mixture of two fluids in a two-dimensional rectangular box is used as a test problem. It is known that the onset of convection is oscillatory below a certain negative value of one of the parameters (the separation ratio), giving rise to very complex dynamics. A nontrivial diagram of periodic orbits is first deployed by varying only a parameter (the Rayleigh number), and some of the bifurcations found on the main branch of periodic orbits are followed by adding a second parameter (the Prandtl number). Several codimension-two points are found, and one of them, a double-Hopf, is studied in more detail to show the robustness and power of the method. Finally, the boundaries of a resonance region (Arnold's tongue) found in [40] are also computed.

The layout of the paper is as follows. As mentioned, section 2 describes Newton-Krylov continuation methods and their application to the computation of cycles, and section 3 describes the extended systems used to track the different codimension-one bifurcations. Section 4 describes the example problem, including the particular form of the variational equations in section 4.1. Section 5 contains the results, starting with the bifurcation diagrams of periodic orbits and invariant tori. Section 5.1 describes the curves of bifurcation, section 5.2 presents results on the resonance region, and section 6 includes information on the performance of the algorithms on the test problem. Finally, the paper concludes with section 7 which includes some remarks on the straightforward extensions to bifurcations of fixed points and to higher codimension cases.

2. Matrix-free continuation methods. Continuation methods allow following one-dimensional manifolds of solutions of a nonlinear system

$$
H(X)=0
$$

starting at an initial $X_{0}$, with $X \in \mathcal{U} \subset \mathbb{R}^{m+1}, H(X) \in \mathbb{R}^{m}$, and $\mathcal{U}$ being the domain of definition of $H$. If $\operatorname{rank}\left(D_{X} H\left(X_{0}\right)\right)=m$, (1) defines, locally, a curve of solutions passing through $X_{0}[20,43]$. To fix ideas, the first $m$ components of $X, \tilde{X}=\left(X_{1}, \ldots, X_{m}\right)$ can be regarded as state variables, and the last as a parameter of the system. Predictor-corrector implementations compute an approximate new solution by extrapolation from previously computed points, which is corrected by different variants of Newton's method. To do so, continuation algorithms add a new equation to system (1), which is usually the equation of a hyperplane passing through the predicted point and with normal vector an approximation of the tangent to the curve of solutions, which can also be approximated from previous points. In this way the hyperplane cuts the manifold of solutions transversely, and the full system defines a unique new set of state variables and the corresponding value of the parameter satisfying (1). Possible implementations differ in the way the extrapolation is performed [43] and in the variant of Newton's method employed. In some cases globalization techniques are used [27] in order to extend the domain of convergence.

In the case of large-scale systems, the direct linear solvers are substituted by iterative methods. Among them, matrix-free methods based on Krylov subspaces are preferred [30, 9]. 
The restarted generalized minimal residual method (GMRES(M)) [32] has always been used in this paper. To solve a linear system $A x=b$, these methods require a subroutine that computes the action by the matrix, $v \rightarrow A v$, and a preconditioning subroutine that solves linear systems with a matrix $\mathcal{M}$, which approximates $A$. The latter subroutine is needed to accelerate the convergence of the linear solvers. Instead of $A x=b$, the left preconditioned system $\mathcal{M}^{-1} A x=\mathcal{M}^{-1} b$, for instance, is solved. To apply $\mathcal{M}^{-1}$ the operator $\mathcal{M}$ must be relatively easy to invert. When equilibria of fluid mechanics or reaction-diffusion problems are computed, the operator $\mathcal{M}$ might correspond to the discretization of only the dissipative part of the equations, since in most cases it is responsible for the bad conditioning of the Jacobian of $H$ with respect to the first $m$ components, $D_{\tilde{X}} H$ (see [45], for instance).

Therefore, a generic continuation code needs the user to provide an initial solution, $X_{0}$, and the following three main subroutines:

- $\mathrm{fun}(\mathrm{X}, \mathrm{h})$, computing the function $h=H(X)$ from $X$;

- $\operatorname{dfun}(\mathrm{X}, \delta \mathrm{x}, \delta \mathrm{h})$, which computes the action by the Jacobian $\delta h=D_{X} H(X) \delta X$ from $X$ and $\delta X ;$ and

- $\operatorname{prec}(\mathrm{X}, \delta \mathrm{Y}, \delta \mathrm{Z})$, which solves $\mathcal{M} \delta Z=\delta Y$ from $X$ and $\delta Y$, with $\mathcal{M}$ being an approximation of $D_{\tilde{X}} H(X)$.

Newton-Krylov continuation codes, including other refinements such as studying the stability of the solutions, bifurcation detection [15], and branch switching at bifurcation points, have been developed by many authors (see [9] and references therein), usually to compute fixed points of large-scale vector fields. By properly defining $H$, other invariant objects can be obtained using the same basic algorithm. The availability of several libraries of linear solvers (see, for instance, $[1,14]$ ) makes the coding of Newton-Krylov continuation programs relatively easy. In the rest of the paper we will assume that one such code is available, and we will only focus on setting the function $H$, specifying the different invariant objects or bifurcation loci, and on how the action by the Jacobian of $H$ can be efficiently computed.

For the kind of problems we will study, the preconditioning subroutine will not be needed, and therefore no further references to prec will be made. The reasons are explained in section 6 .

2.1. Continuation of periodic orbits. We explain here, for completeness, how to use the previous techniques for the computation of periodic orbits by Newton-Krylov methods. The changes required to go one step forward and compute their bifurcation loci will then be easier to follow. Since their introduction in [39], these methods have been applied by several authors for the computation of limit cycles in different problems [48, 28, 49, 51, 50, 52, 17]. Poincaré sections were used in [39], but in this paper they are avoided just to simplify the formulation of the extended systems for the loci of bifurcations in the next section.

Consider a high-dimensional autonomous system of dissipative ODEs,

$$
\dot{y}=f(y, p), \quad(y, p) \in \mathcal{V} \subset \mathbb{R}^{n} \times \mathbb{R}^{k},
$$

with $\mathcal{V}$ being the domain of definition of $f$. We assume, for the moment, that it depends on a single distinguished parameter $p(k=1)$. The system could have been obtained, as in section 4 , after the spatial truncation of a system of parabolic PDEs. Let $\varphi(t, x, p)$ be its 
solution with initial condition $x$ at $t=0$ for a fixed $p$. It satisfies

$$
D_{t} \varphi(t, x, p)=f(\varphi(t, x, p), p) \text { and } \varphi(0, x, p)=x .
$$

A one-dimensional manifold of periodic orbits of (2) (more precisely, a point of each cycle) is given by the system

$$
H(X)=\left(\begin{array}{c}
x-\varphi(T, x, p) \\
g(x)
\end{array}\right)=0
$$

with $X=(x, T, p)$, and where $g(x)=0$ is a phase condition selecting a single point on the orbit. For simplicity we use the equation of a hyperplane, cutting transversely the periodic orbit, of the form $g(x)=v_{\pi}^{\top}\left(x-x^{\pi}\right)=0$, passing through a point $x^{\pi}$ and with normal vector $v_{\pi}$. Each solution of (4) obtained during a continuation process provides a point on the periodic orbit $x$, its period $T$, and the value of the parameter $p$ corresponding to the orbit.

For this particular invariant object, the subroutines fun and df un of a generic continuation code have to work as follows. If $X=(x, T, p)$, $\mathrm{fun}(\mathrm{X}, \mathrm{h})$ has to integrate the system (2) $T$ units of time, with initial condition $y(0)=x$, to compute $x-\varphi(T, x, p)$, with $T$ being the approximate period contained in $X$. It also has to evaluate $g(x)$. If $X=(x, T, p)$ and $\delta X=(\delta x, \delta T, \delta p), \operatorname{dfun}(\mathrm{X}, \delta \mathrm{x}, \delta \mathrm{h})$ must compute the action of the Jacobian $D_{X} H$ on the vector $\delta X$, i.e.,

$$
D_{X} H(X) \delta X=\left(\begin{array}{c}
\delta x-D_{t} \varphi(T, x, p) \delta T-D_{x} \varphi(T, x, p) \delta x-D_{p} \varphi(T, x, p) \delta p \\
D_{x} g(x) \delta x
\end{array}\right) .
$$

For this purpose let us define

$$
\begin{aligned}
y(t) & =\varphi(t, x, p), \\
y_{1}(t) & =D_{x} \varphi(t, x, p) \delta x+D_{p} \varphi(t, x, p) \delta p .
\end{aligned}
$$

Since $\varphi(t, x, p)$ is the solution of (2), i.e., (3) holds, then $D_{t} \varphi(T, x, p)=f(y(T), p)=\dot{y}(T)$. In addition, by taking time derivatives of (6) and (7), the system

$$
\begin{aligned}
& \dot{y}=f(y, p), \\
& \dot{y_{1}}=D_{y} f(y, p) y_{1}+D_{p} f(y, p) \delta p
\end{aligned}
$$

is obtained for $y(t)$ and $y_{1}(t)$. Moreover, $y(0)=x$, and since $\varphi(0, x, p)=x$ for any $p$, $D_{x} \varphi(0, x, p)=I$ and $D_{p} \varphi(0, x, p)=0$. Then $y_{1}(0)=D_{x} \varphi(0, x, p) \delta x+D_{p} \varphi(0, x, p) \delta p=\delta x$.

Summarizing, dfun must integrate (8) and (9) from $t=0$ to $t=T$, with initial conditions $y(0)=x$ and $y_{1}(0)=\delta x$, for the values of $T, p$, and $\delta p$ contained in $X$ and $\delta X$. Then

$$
D_{X} H(X) \delta X=\left(\begin{array}{c}
\delta x-\dot{y}(T) \delta T-y_{1}(T) \\
D_{x} g(x) \delta x
\end{array}\right) .
$$

Equation (9) is a first order variational equation obtained by linearizing (8) about $y(t)$.

These calculations imply the integration of systems of at most $2 n$ equations. The bifurcation diagrams of periodic orbits in section 5 were obtained in this way. 
It has been supposed that equations (4) completely determine a unique periodic orbit. Some problems may require additional conditions. For instance, in systems with translational or rotational invariances in periodic domains, the spatial phases must be specified (see [48, 35]). This can be solved by fixing some components of the state vector $x$ or, in general, by adding new phase conditions like that used to fix the translational time invariance $(g(x)=0)$. Their inclusion does not modify the above setup.

3. Continuation of bifurcation curves. Suppose now that the system (2) depends on two distinguished parameters $p=\left(p_{1}, p_{2}\right)(k=2)$. We are interested in tracking curves of codimension-one bifurcations of periodic orbits in systems with or without symmetries. Therefore, we must define, for each particular kind of bifurcation, the corresponding extended system $H(X)=0$. The augmented systems are described in the following subsections. The case of turning points (saddle-nodes) or period-doubling bifurcations is treated in more detail to show how to deal with the computation of the action by the Jacobian, which involves the integration of second order variational equations.

3.1. Saddle-node and period-doubling bifurcations. The saddle-node and perioddoubling bifurcations of periodic orbits can be treated simultaneously by means of a variant of the formulation in $[42,26]$. It was originally studied for fixed points of ODEs, and it is adapted here for the singularities of the equation $x-\varphi(t, x, p)=0$. The two types of bifurcation points are solutions of the system $H(x, u, T, p)=0$ given by

$$
\begin{aligned}
x-\varphi(T, x, p) & =0, \\
g(x) & =0, \\
\lambda u-\left(D_{x} \varphi(T, x, p) u-\frac{1}{2}(1+\lambda) \frac{\langle w, u\rangle}{\langle w, w\rangle} w\right) & =0, \\
\left\langle u_{r}, u\right\rangle & =1,
\end{aligned}
$$

with $w=f(x, p)$ and $\langle\cdot, \cdot\rangle$ being the Euclidean dot product. The two cases are distinguished by setting $\lambda=+1$ and $\lambda=-1$, respectively. The first two equations are the same as in (4). They define a single point on a periodic orbit of (2), satisfying the phase condition $g(x)=0$.

In the case of saddle-node bifurcations $(\lambda=+1)$ the third equation becomes

$$
D_{x} \varphi(T, x, p) u-\frac{\langle w, u\rangle}{\langle w, w\rangle} w=u
$$

which states that there is an eigenvector $u$ corresponding to the eigenvalue +1 of $D_{x} \varphi(T, x, p)$ orthogonal to the trivial $w=f(x, p)$. This is the main difference from the classical formulation in [26]. The Wieland's deflation term (see [30]), $(\langle w, u\rangle /\langle w, w\rangle) w$, shifts the trivial +1 to zero. This guarantees the regularity of the global system (11)-(14).

For period-doubling bifurcations $(\lambda=-1)$ the third equation is $D_{x} \varphi(T, x, p) u=-u$, stating that there is an eigenvector $u$ corresponding to the eigenvalue -1 of $D_{x} \varphi(T, x, p)$.

Finally, $\left\langle u_{r}, u\right\rangle=1$ fixes the undetermined multiplicative constant of the eigenvector, with $u_{r}$ being a reference vector. We have used $u_{r}=u$ in the computations of section 5 , changing the derivatives of this equation accordingly. 
The extended system (11)-(14) fits into the general framework of Newton-Krylov methods presented in section 2 , with $X=(x, u, T, p)$ a vector of unknowns of dimension $m+1=2 n+3$, and $H(x, u, T, p)=0$ a system of $m=2 n+2$ equations. This defines, locally, a one-dimensional manifold of codimension-one bifurcations, close to any initial solution $X_{0}$.

In order to obtain $H(x, u, T, p)$, we proceed as in section 2.1. Let us define

$$
\begin{aligned}
y(t) & =\varphi(t, x, p), \\
y_{1}(t) & =D_{x} \varphi(t, x, p) u,
\end{aligned}
$$

which satisfy

$$
\begin{aligned}
\dot{y} & =f(y, p), & & y(0)=x, \\
\dot{y}_{1} & =D_{y} f(y, p) y_{1}, & & y_{1}(0)=u .
\end{aligned}
$$

By integrating these equations a time $T, \varphi(T, x, p)=y(T)$ and $D_{x} \varphi(T, x, p) u=y_{1}(T)$ are obtained. The computation of the deflation term and (12) and (14) is straightforward. The corresponding subroutine fun would be similar to the dfun for the computation of periodic orbits, except for the deflation term and (14), which must be added.

The action of $D_{X} H(x, u, T, p)$ on $(\delta x, \delta u, \delta T, \delta p)$ is

$$
\begin{aligned}
& \delta x-D_{t} \varphi(T, x, p) \delta T-D_{x} \varphi(T, x, p) \delta x-D_{p} \varphi(T, x, p) \delta p \\
& D g(x) \delta x \\
& \lambda \delta u-D_{t x}^{2} \varphi(T, x, p)(u, \delta T)-D_{x x}^{2} \varphi(T, x, p)(u, \delta x)-D_{x p}^{2} \varphi(T, x, p)(u, \delta p) \\
& -D_{x} \varphi(T, x, p) \delta u \\
& +\frac{1+\lambda}{2\langle w, w\rangle}\left(\langle w, u\rangle z+\left(\langle z, u\rangle+\langle w, \delta u\rangle-\frac{2\langle w, z\rangle}{\langle w, w\rangle}\langle w, u\rangle\right) w\right), \\
& \left\langle u_{r}, \delta u\right\rangle
\end{aligned}
$$

where $w=f(x, p)$ and $z=D_{x} f(x, p) \delta x+D_{p} f(x, p) \delta p$.

In order to compute the different terms in (15) and (17) let us define

$$
\begin{aligned}
y(t) & =\varphi(t, x, p), \\
y_{1}(t) & =D_{x} \varphi(t, x, p) u, \\
y_{2}(t) & =D_{x} \varphi(t, x, p) \delta x+D_{p} \varphi(t, x, p) \delta p, \\
y_{3}(t) & =D_{x x}^{2} \varphi(t, x, p)(u, \delta x)+D_{x p}^{2} \varphi(t, x, p)(u, \delta p), \\
y_{4}(t) & =D_{x} \varphi(t, x, p) \delta u .
\end{aligned}
$$

The vectors $y_{i}(T), i=1, \ldots, 4$, contain the required derivatives of $\varphi(T, x, p)$ and $D_{x} \varphi(T, x, p) u$ with respect to $x$ and $p$. By taking time derivatives like in the case of the periodic orbits, it 
is not difficult to see that the system which must be integrated to obtain them is

$$
\begin{aligned}
\dot{y} & =f(y, p), & y(0) & =x, \\
\dot{y}_{1} & =D_{y} f(y, p) y_{1}, & y_{1}(0) & =u, \\
\dot{y}_{2} & =D_{y} f(y, p) y_{2}+D_{p} f(y, p) \delta p, & y_{2}(0) & =\delta x \\
\dot{y}_{3} & =D_{y} f(y, p) y_{3}+D_{y y}^{2} f(y, p)\left(y_{1}, y_{2}\right)+D_{y p}^{2} f(y, p)\left(y_{1}, \delta p\right), & y_{3}(0) & =0 \\
\dot{y}_{4} & =D_{y} f(y, p) y_{4}, & y_{4}(0) & =\delta u .
\end{aligned}
$$

Finally, the second term of (15) is computed as before, and that of (17) needs only the evaluation of the action by $D_{y} f(y(T), p)$, i.e.,

$$
\begin{aligned}
D_{t} \varphi(T, x, p) \delta T & =f(y(T), p) \delta T=\dot{y}(T) \delta T, \\
D_{t x}^{2} \varphi(T, x, p)(u, \delta T) & =\delta T D_{y} f(\varphi(T, x, p), p) D_{x} \varphi(T, x, p) u=\delta T D_{y} f(y(T), p) y_{1}(T) .
\end{aligned}
$$

Obtaining the rest of the terms in (17) is straightforward, requiring also derivatives of $f(y, p)$.

The difference between systems (8)-(9) and (24)-(28) is the presence of two new first order variational equations, (25) and (28), and one second order variational equation, (27). If a subroutine dfun for cycles is already available, it could be completed for this new task.

Summarizing, the evaluation of $H(X)$ requires the integration of a system of ODEs of dimension $2 n$, and that of $D_{X} H(X) \delta X$ requires the integration of a system of dimension $5 n$. Since (28) is only coupled with (24), it is possible to separate the system into two subsystems, which can be solved sequentially or in parallel. Equations (24)-(27) form a coupled triangular system of ODEs, with a total dimension $4 n$, which provide $y, y_{1}, y_{2}$, and $y_{3}$. Equations (24) and (28), with total dimension $2 n$, must be solved together to obtain $y_{4}$.

It is important to notice that, since the way $D_{X} H(X) \delta X$ is computed gives the exact action of the Jacobian $D_{X} H(X)$, the quadratic convergence of Newton's method can be preserved. This is an essential ingredient for efficiency, and it is what happens in the example presented below. The approximation of $D_{X} H(X) \delta X$ by finite differences usually leads to a slower convergence, if any.

3.2. Neimark-Sacker bifurcations. A Neimark-Sacker bifurcation point of a periodic orbit, with critical multiplier $e^{i \theta}$ and associate eigenvector $u+i v$, is a solution of the system $H(x, u, v, T, \theta, p)=0$, defined in [18] for fixed points of ODEs and in [21] for that of maps, and modified here to include the phase condition. It is given by

$$
\begin{aligned}
x-\varphi(T, x, p) & =0, \\
g(x) & =0, \\
u \cos \theta-v \sin \theta-D_{x} \varphi(T, x, p) u & =0, \\
u \sin \theta+v \cos \theta-D_{x} \varphi(T, x, p) v & =0, \\
\langle u, u\rangle+\langle v, v\rangle & =1, \\
\langle u, v\rangle & =0 .
\end{aligned}
$$

The first two equations define again a single point on a periodic orbit of (2), satisfying the phase condition $g(x)=0$. The third and fourth are obtained by separating real and imaginary 
parts of $D_{x} \varphi(T, x, p)(u+i v)=e^{i \theta}(u+i v)$, and the last two uniquely determine the eigenvector. The vector of unknowns $X=(x, u, v, T, \theta, p)$ has dimension $m+1=3 n+4$, and the $m=$ $3 n+3$ equations define the curve of Neimark-Sacker bifurcation points. The work required to evaluate $H(X)$ and $D_{X} H(X) \delta X$ doubles since there are now two products by $D_{x} \varphi(T, x, p)$ in the system. They can obviously be computed in parallel. All the terms required to compute $H$ or the action by its Jacobian have already been considered in the previous subsection.

3.3. Pitchfork bifurcations. Suppose now that the initial system is $\mathcal{T}$-invariant, i.e., $f(\mathcal{T} x, p)=\mathcal{T} f(x, p)$, with $\mathcal{T}$ a linear transformation verifying $\mathcal{T}^{2}=I$, and that we are looking for symmetry-breaking bifurcations of fixed cycles satisfying $\mathcal{T} x(t)=x(t) \forall t$. Two possible extended systems are available in the literature to follow the pitchfork bifurcations. The first consists in using the same system as that for the saddle-node bifurcation, but restricting $x$ to the subspace of $\mathcal{T}$-symmetric vectors and $u$ to that of $\mathcal{T}$-antisymmetric vectors [53]. To benefit from the reduced dimension of the system to be solved, the time integration should be able to work on symmetric or antisymmetric subspaces. Another possibility, which is only a slight modification of the saddle-node system, was introduced in [16] for the pitchfork bifurcations of fixed points of ODEs. For periodic orbits, a deflation term must be added, as in the case of saddle-node points, to avoid the singularity of the system due to the presence of the trivial +1 multiplier. Let $H(x, u, T, \xi, p)=0$ be

$$
\begin{aligned}
x-\varphi(T, x, p)+\xi \phi & =0, \\
g(x) & =0, \\
\langle x, \phi\rangle & =0, \\
u-\left(D_{x} \varphi(T, x, p) u-\frac{\langle w, u\rangle}{\langle w, w\rangle} w\right) & =0, \\
\left\langle u_{r}, u\right\rangle & =1,
\end{aligned}
$$

with $w=f(x, p)$. The slack variable $\xi$ and the third equation are introduced to make the system regular, with $\phi$ being a given antisymmetric vector, $\mathcal{T} \phi=-\phi$. At the solution $\xi=0$. See [16] for details. Now $X=(x, u, T, \xi, p)$ has dimension $m+1=2 n+4$, and the $m=2 n+3$ equations define the curve of pitchfork points.

In the case of symmetric cycles, i.e., when $\mathcal{T} \varphi(T / 2, x, p)=x$, the same methods can be used but the time required to integrate the systems is reduced by half.

All of the above extended systems can also be written in terms of a Poincaré map. Then the deflation term is not needed, and $T$ is obtained as a by-product (the time needed to come back to the initial point at the last iteration of Newton's method), but then some kind of interpolation must be included to compute the intersection of the trajectories with the section, and the action of the derivatives of the map $\varphi(t, x, p)$ involves projections onto the section $[44,23,38]$. In any case this is a second option.

The bifurcations of steady solutions can also be continued with the preceding systems by taking as $T$ a characteristic time of the problem, which is no longer an unknown, and removing the phase condition. This is not, in general, the most efficient method because it involves time integrations, but it prevents the need for finding adequate preconditioners for the standard extended systems. 
4. Thermal convection in binary fluid mixtures. The thermal convection of a binary mixture, filling a two-dimensional rectangular domain $\Omega$ heated from below, has been used as a test problem. It is not trivial and gives rise to a very rich dynamics with all the types of bifurcations we are interested in continuing, and, in addition, since it was also used in [40,37], we had already located some bifurcation points, which are taken here as initial conditions to follow the curves of bifurcations.

The equations of the system are the conservation of mass, momentum, and energy and that for the evolution of one of the concentrations (the denser in this case) [6]. The units used to write them in nondimensional form are the height of the domain $h$, the thermal diffusion time $h^{2} / \kappa$, where $\kappa$ is the thermal diffusivity, the temperature difference, $\Delta T$, between the top and bottom sides, and $\bar{C}(\bar{C}-1) D^{\prime} \Delta T / D$, where $\bar{C}$ is the volume-average concentration, $D>0$ the mass diffusion coefficient, and $D^{\prime}$ the thermal diffusion coefficient. In nondimensional units $\Omega=[0, \Gamma] \times[0,1]$, where $\Gamma$ is the width $l$ to the height ratio, and now $x$ and $y$ are the horizontal and vertical coordinates, respectively.

The basic conductive and linearly stratified state, which is a solution of the equations for any value of the parameters, is given by zero velocity $\mathbf{v}_{b}=0$, and nondimensional temperature $T_{b}=T_{b}(0)-y$ and concentration $C_{b}=C_{b}(0)-y$ linear profiles. The values $T_{b}(0)$ and $C_{b}(0)$ are related constants because of the boundary conditions defined below. The Boussinesq approximation of the equations for the perturbation $(\mathbf{v}, \Theta, \Sigma)$, of the basic state $\left(\mathbf{v}_{b}, T_{b}, C_{b}\right)$, is written in terms of a stream-function, $\psi$, i.e., $\mathbf{v}=\left(-\partial_{y} \psi, \partial_{x} \psi\right)$, and an auxiliary function $\eta=\Sigma-\Theta$. They are

$$
\begin{aligned}
& \partial_{t} \nabla^{2} \psi+J\left(\psi, \nabla^{2} \psi\right)=\sigma \nabla^{4} \psi+\sigma R a\left[(S+1) \partial_{x} \Theta+S \partial_{x} \eta\right], \\
& \partial_{t} \Theta+J(\psi, \Theta)=\nabla^{2} \Theta+\partial_{x} \psi, \\
& \partial_{t} \eta+J(\psi, \eta)=L \nabla^{2} \eta-\nabla^{2} \Theta
\end{aligned}
$$

with $J(f, g)=\partial_{x} f \partial_{y} g-\partial_{y} f \partial_{x} g$. No-slip $(\mathbf{v}=0$ on $\partial \Omega)$ and nonporous $\left(\partial_{n}(\Sigma-\Theta)=0\right.$ on $\left.\partial \Omega\right)$ conditions at the boundaries become $\psi=\partial_{n} \psi=\partial_{n} \eta=0$, and constant temperature at top and bottom and insulating lateral sides, in terms of the temperature perturbation, are $\Theta=0$ on $y=0,1$ and $\partial_{x} \Theta=0$ on $x=0, \Gamma$. Notice that in this way the incompressibility condition is identically fulfilled, the boundary conditions for $\Theta$ and $\Sigma$ decouple, and the number of unknowns is reduced.

The problem depends on the aspect ratio, $\Gamma$, the nondimensional Rayleigh, $R a$, Prandtl, $\sigma$, and Lewis, $L$, numbers, and the separation ratio, $S$, defined as

$$
\Gamma=\frac{l}{h}, \quad R a=\frac{\alpha g \Delta T h^{3}}{\kappa \nu}, \quad \sigma=\frac{\nu}{\kappa}, \quad L=\frac{D}{\kappa}, \quad S=\frac{\bar{C}(1-\bar{C}) \beta D^{\prime}}{\alpha D},
$$

respectively. In the definitions of the parameters $\nu$ means the kinematic viscosity, and $\alpha$ and $\beta$ (taken positive) are the thermal and compositional expansion coefficients, respectively.

In the continuation experiments of bifurcation points we fix $\Gamma=4, L=0.03$, and $S=$ -0.1 . The last two values correspond to a mixture of two isotopes of helium in liquid state. According to the definition of $S, D^{\prime}<0$, and initially the concentration gradient is stabilizing in opposition to the destabilizing temperature gradient. If $S$ is below a negative critical value, as it is in the test problem, the primary bifurcation from the basic state is a Hopf bifurcation. 
The group of symmetries of this system is $\mathbb{Z}_{2} \times \mathbb{Z}_{2}$ generated by the reflections $R_{x}$ and $R_{y}$, with respect to the vertical and horizontal midplanes; i.e., changing $x$ by $\Gamma-x$ and the sign of $\psi$, or changing $y$ by $1-y$ and the sign of all three functions, leaves the system invariant. These symmetries give rise to pitchfork bifurcations of fixed points, periodic orbits, and even of invariant tori as sets.

To obtain the numerical solutions, the functions $\psi, \Theta$, and $\eta$ are approximated by a pseudospectral method. Collocation on a mesh of $n_{x} \times n_{y}=64 \times 16$ Gauss-Lobatto points has been used in all of the calculations shown. This gives $n=3072$ unknowns. The mesh is enough to have a good accuracy in the range of parameters considered because the solutions are quite smooth. Finer resolutions $n_{x} \times n_{y}=96 \times 24$ were used to check the results and, for instance, the relative difference between the leading multipliers at the double-Hopf bifurcation shown below is less than $10^{-6}$. The stiff system of ODEs obtained after the spatial discretization is integrated by using BDF-extrapolation formulas, as described, for instance, in [40]. Other details on the physical problem can also be found there.

4.1. Variational equations. In the following sections the two continuation parameters will be $R a$ and $\sigma$. Then the terms $J(\cdot, \cdot), \sigma \Delta^{2} \psi$, and $\sigma R a\left[(S+1) \partial_{x} \Theta+S \partial_{x} \eta\right]$ in (40)-(42) are quadratic or cubic in the unknowns $(\psi, \Theta, \eta, \sigma$, and $R a)$, and the variational equations are easily computed. Now (25)-(27) become

$$
\begin{aligned}
\partial_{t} \Delta \psi_{1}+J\left(\psi, \Delta \psi_{1}\right)+J\left(\psi_{1}, \Delta \psi\right) & =\sigma \Delta^{2} \psi_{1}+\sigma R a\left[(S+1) \partial_{x} \Theta_{1}+S \partial_{x} \eta_{1}\right], \\
\partial_{t} \Theta_{1}+J\left(\psi, \Theta_{1}\right)+J\left(\psi_{1}, \Theta\right) & =\Delta \Theta_{1}+\partial_{x} \psi_{1}, \\
\partial_{t} \eta_{1}+J\left(\psi, \eta_{1}\right)+J\left(\psi_{1}, \eta\right)=L \Delta \eta_{1}-\Delta \Theta_{1}, & \\
\partial_{t} \Delta \psi_{2}+J\left(\psi, \Delta \psi_{2}\right)+J\left(\psi_{2}, \Delta \psi\right)= & \sigma \Delta^{2} \psi_{2}+\sigma R a\left[(S+1) \partial_{x} \Theta_{2}+S \partial_{x} \eta_{2}\right]+\delta \sigma \Delta^{2} \psi \\
& +(\sigma \delta R a+\delta \sigma R a)\left[(S+1) \partial_{x} \Theta+S \partial_{x} \eta\right], \\
\partial_{t} \Theta_{2}+J\left(\psi, \Theta_{2}\right)+J\left(\psi_{2}, \Theta\right)= & \Delta \Theta_{2}+\partial_{x} \psi_{2}, \\
\partial_{t} \eta_{2}+J\left(\psi, \eta_{2}\right)+J\left(\psi_{2}, \eta\right)= & L \Delta \eta_{2}-\Delta \Theta_{2},
\end{aligned}
$$

and

$$
\begin{aligned}
\partial_{t} \Delta \psi_{3}+J\left(\psi, \Delta \psi_{3}\right)+J\left(\psi_{3}, \Delta \psi\right)= & \sigma \Delta^{2} \psi_{3}+\sigma R a\left[(S+1) \partial_{x} \Theta_{3}+S \partial_{x} \eta_{3}\right]+\delta \sigma \Delta^{2} \psi_{1} \\
& +(\sigma \delta R a+\delta \sigma R a)\left[(S+1) \partial_{x} \Theta_{1}+S \partial_{x} \eta_{1}\right] \\
& -J\left(\psi_{1}, \Delta \psi_{2}\right)-J\left(\psi_{2}, \Delta \psi_{1}\right), \\
\partial_{t} \Theta_{3}+J\left(\psi, \Theta_{3}\right)+J\left(\psi_{3}, \Theta\right)= & \Delta \Theta_{3}+\partial_{x} \psi_{3}-J\left(\psi_{1}, \Theta_{2}\right)-J\left(\psi_{2}, \Theta_{1}\right), \\
\partial_{t} \eta_{3}+J\left(\psi, \eta_{3}\right)+J\left(\psi_{3}, \eta\right)= & L \Delta \eta_{3}-\Delta \Theta_{3}-J\left(\psi_{1}, \eta_{2}\right)-J\left(\psi_{2}, \eta_{1}\right),
\end{aligned}
$$

respectively, and (28) is like the first. They differ only in some forcing terms which have the same structure as those in the original equations. Therefore, any time integrator for the initial system can be easily adapted to integrate these equations.

The simplicity in finding the variational equations up to order two (or even to higher orders) is common for many problems in fluid mechanics and other branches of mathematical physics governed by equations with linear diffusion and polynomial nonlinear terms. 

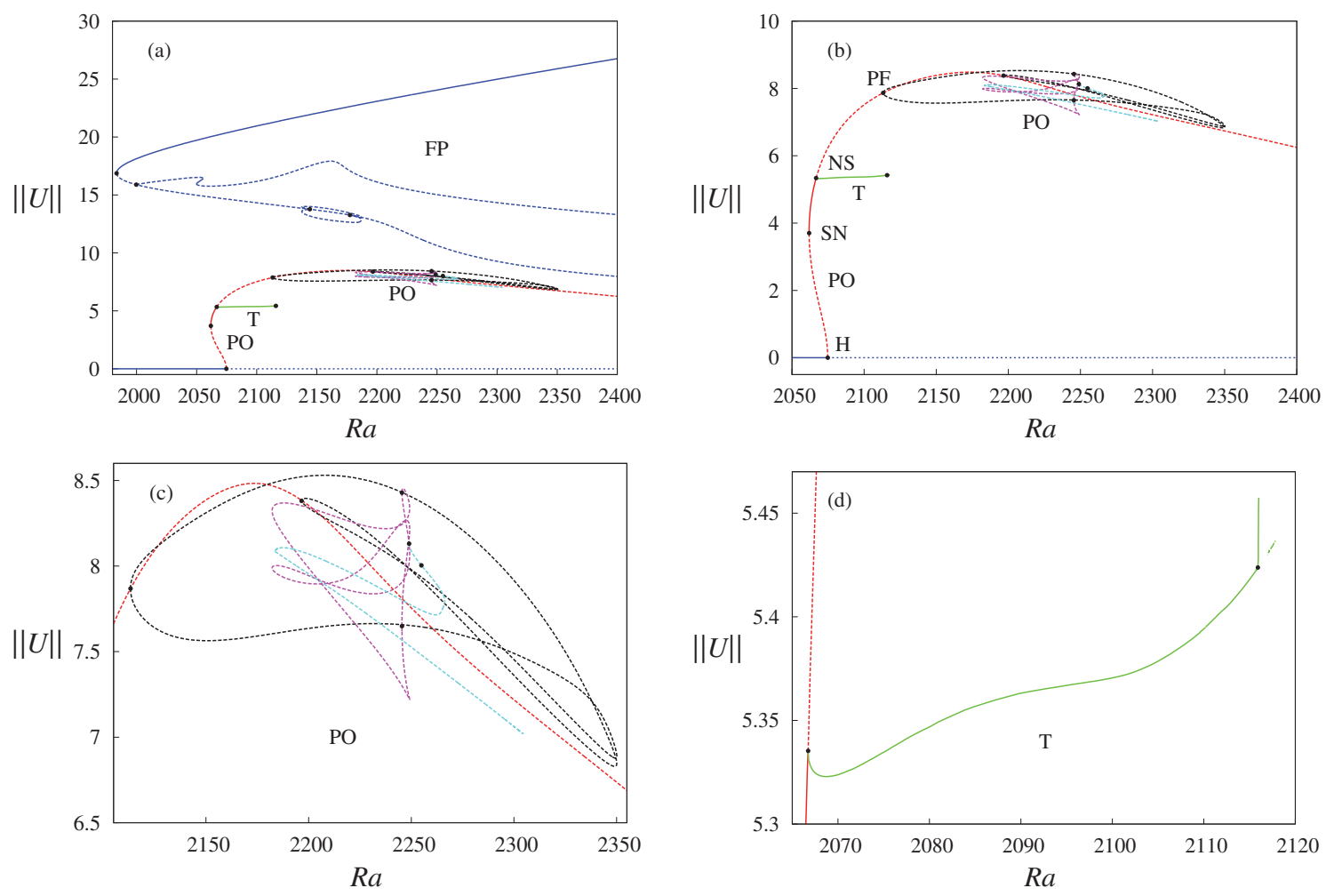

Figure 1. Bifurcation diagrams for $\sigma=0.6$.

The time-stepping method and time-step size used for the coupled system (24)-(28) are the same as those used for the initial equation (24) of the test problem. This has been the case every time we have added first order variational equations in several fluid mechanics problems, and is the case here when including those of second order. Only the diffusion terms were taken implicitly, and therefore a trivial block diagonal system had to be solved at each time step.

5. Results. To show the origin of the initial conditions for the continuation of the bifurcation curves, we present the bifurcation diagram of fixed points, periodic orbits, and invariant tori for $\sigma=0.6$ in Figure 1. This value was used in [36, 40, 37] to provide examples of the continuation of periodic orbits by multiple shooting, and of invariant tori with two different algorithms, so the main branch of periodic orbits and that of invariant tori were already known. The Euclidean norm of the vector $U=\left(\psi_{i j}, \Theta_{i j}, \eta_{i j}\right)$, containing the values of the three functions $\psi, \Theta$, and $\eta$ at the mesh of inner collocation points, is plotted versus $R a$. In the case of periodic orbits it corresponds to the norm of one of their intersections with the hyperplane $\psi_{38,9}=0$, where the subindex refers to the indices of the collocation mesh, and for the tori it refers to their intersections with $\psi_{38,9}=0$ and $\Theta_{58,8}=0$. Solid and dashed lines mean stable and unstable solutions, respectively. The curve $\psi=0$, separating clockwise and counterclockwise rotating vortices, sweeps the full rectangular domain $\Omega$ in a period. Therefore all of these periodic orbits cut transversely the surface $\psi(x, y)=0$ for any $(x, y) \in \Omega$. 
The point $\left(x_{38}, y_{9}\right)$, close to the horizontal midplane $y=1 / 2$ and to $x=2.5$, was selected. In this way it was not necessary to change the phase condition during these calculations.

The upper branches in Figure 1(a) (in blue) correspond to fixed points (FP). They appear at a saddle-node bifurcation at $R a=1983.40$. The upper part is stable, and three pitchfork bifurcations can be seen along the unstable lower branch. A small stable portion, limited by two saddle-node points, can be observed in the middle branch of fixed points. The steady states extend below the critical value given by the linear stability of the basic conductive state $\|U\|=0$ (blue horizontal lower line).

Figures 1(b) and (c) are details of branches of periodic orbits (PO). The conduction state loses stability at $R a=2074.76$ at a subcritical Hopf bifurcation $(\mathrm{H})$. The periodic orbits emerging here (in red) become stable at a saddle-node point (SN) at $R a=2061.85$ and lose stability at $R a=2066.74$ at a Neimark-Sacker (NS) bifurcation. The solutions on this main branch of periodic orbits are always symmetric with respect to the vertical midplane and are also symmetric cycles with respect to the horizontal midplane. So they have, as sets, the same symmetry group $\mathbb{Z}_{2} \times \mathbb{Z}_{2}$ as the equations. By following this curve, we have a first pitchfork bifurcation $(\mathrm{PF})$ which breaks the vertical reflection symmetry. The two new branches (in black) join again at another pitchfork bifurcation on the main branch. The solutions on these new branches are still symmetric cycles with respect to the horizontal midplane. There are secondary pitchfork bifurcations on them, that break the remaining symmetry. Finally, two period-doubling bifurcations were found, one on the new branch without any symmetry (in magenta) and the other on the period-doubled branch (in cyan). The three unstable Floquet multipliers at the end of the latter curve are $2.23 \times 10^{7},-1.50 \times 10^{6}$, and 51.9. It was not possible to continue beyond this point by using simple shooting.

A detail of the branches of tori (T) (in green) is given in Figure 1(d). The main branch starts at $R a=2066.74$ and is stable up to a pitchfork bifurcation at $R a=2115.92$. The rotation number $\rho$ decreases along this curve when $R a$ is increased, starting near, but below, $1 / 6$ and ending close to $1 / 8$ with a $1 / 7$-resonance in $2102.79<R a<2102.80$. One of the two stable branches of tori after the pitchfork bifurcation was continued up to a $1 / 8$-resonance in $2116.18<R a<2116.20$. The periodic orbits inside this region were also computed by continuation methods (see below). Details on the final breakdown of the tori at $R a \approx 2115.92$ can be found in [40].

5.1. Curves of bifurcations. Figure 2(a) shows the bifurcation curves computed by the methods described in section 3. The norm of the intersection of the periodic orbits with the hyperplane used as phase condition, $\psi_{38,9}=0$, is plotted against the two parameters $\sigma$ and Ra. The sections at constant $\sigma=0.1,0.2,0.4,0.6,1,1.5,2.0$, and 2.5 (in red) represent periodic orbits computed by continuation with respect to $R a$. The curve for $\sigma=0.6$ is that shown in Figure 1(b), with the Hopf, saddle-node, Neimark-Sacker, and pitchfork bifurcation points labeled as H, SN, NS, and PF, respectively. They were used as initial conditions to start the bifurcation curves up and down in $\sigma$, which was selected as the distinguished continuation parameter. The linear stability analysis at these points provided the initial eigenvectors. We have not followed period-doubling curves because the system (11)-(14) needed to obtain them is the same as for the saddle-nodes, without the deflation term. Although the maximum value of $\sigma$ in the plots is $\sigma=3$, some curves were calculated beyond this limit. 

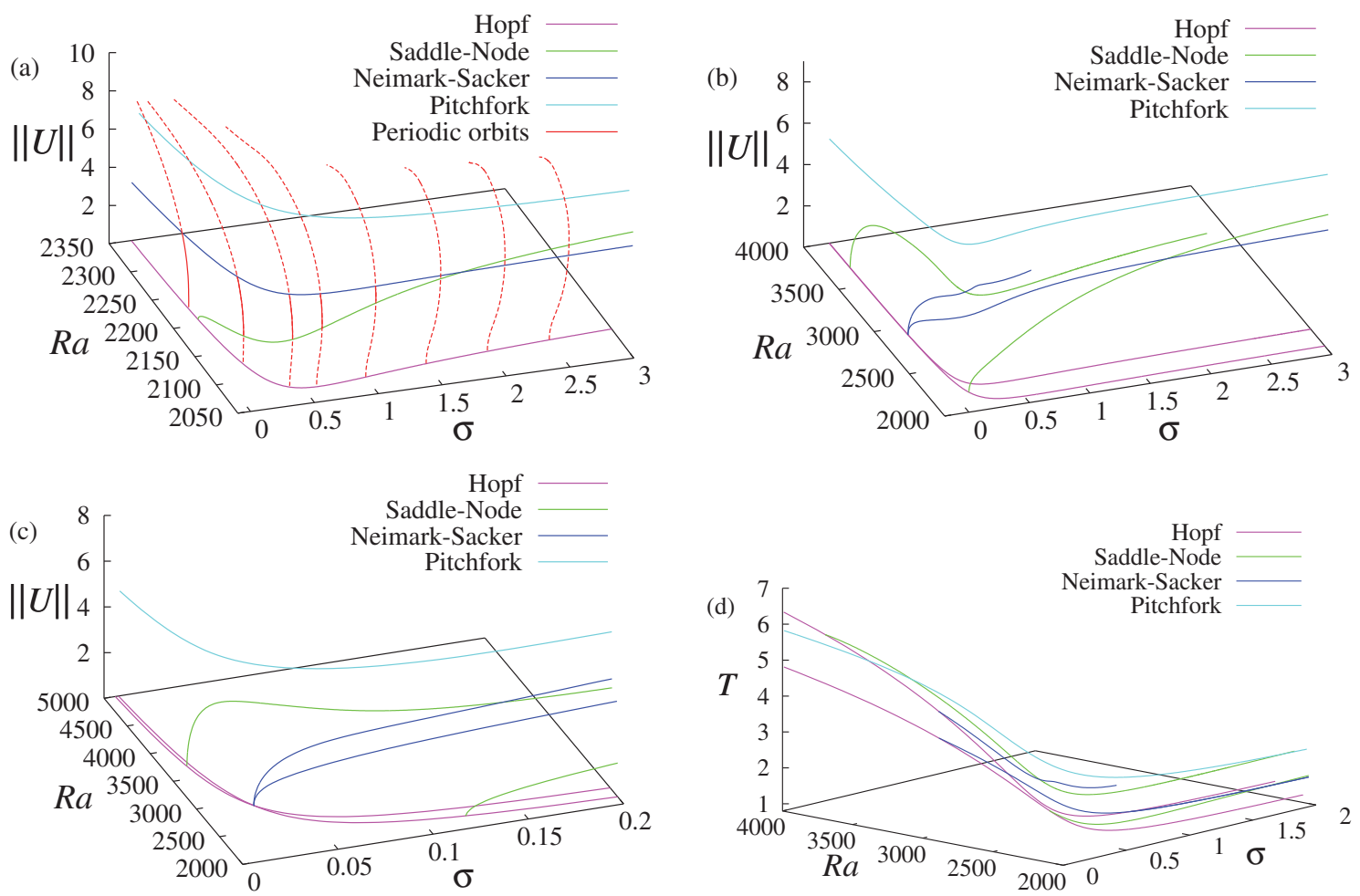

Figure 2. Curves of bifurcation points and period of the corresponding periodic orbits.

The surface $\|U\|=0$ in Figure 2(a) represents the conduction state $\psi=0, \Theta=0$, and $\eta=0$. The curve on this surface (in magenta) is that of Hopf bifurcations giving rise to a first family of periodic orbits. It was computed, as mentioned before, by using the extended system (29)-(34), but in this case $T$ was taken as a fixed value close to the period of the periodic orbits near the Hopf bifurcation for $\sigma=0.6$, and it was not necessary to use the two first equations (29) and (30), since the conduction state is a known trivial solution.

The curve of saddle-nodes (in green) starts at $\sigma=0.122, R a=2209.42$ on the Hopf locus at a Bautin (generalized Hopf) point, which separates the supercritical $(\sigma<0.122)$ from the subcritical $(\sigma>0.122)$ bifurcations.

The curve of Neimark-Sacker points (in blue) provides another boundary for the stability of this first set of periodic orbits. As can be seen in Figure 2(a), they are only stable in the region limited by the Hopf, saddle-node, and Neimark-Sacker curves. The last two cross at $\sigma=1.699$ and $R a=2052.79$ at a fold-Hopf bifurcation of periodic orbits.

A movie (98101_01.gif [local/web 8.51MB]) showing the physical behavior of a cycle for $\sigma=$ 0.5029 and $R a=2072.86$, on the Neimark-Sacker locus, has been included as supplementary material. It is representative of the first set of periodic orbits. The three contour plots correspond from top to bottom to the stream-function $\psi$, the temperature $T=T_{b}+\Theta$, and the concentration $C=C_{b}+\Sigma=C_{b}+\eta+\Theta$. It can be seen, by looking at $\psi$, that it is a fixed cycle with respect to the transformation $R_{x}$ and is a symmetric cycle with respect to $R_{y}$. For the other two functions it must be taken into account that the conduction state has been 
added. The oscillatory regime takes essentially the form of a standing wave. The intensity of the vortices and the amplitude of the oscillations of the temperature and concentration are larger at the two central vortices than at the lateral. The sense of rotation of the vortices changes sign twice each period, and there is a marked phase lag between the temperature and concentration oscillations.

The locus of pitchfork bifurcations (in cyan) was calculated down to $\sigma=3.960 \times 10^{-2}$, where the Rayleigh number was $R a=47825$, and up to $\sigma=6$ to show the performance of the methods in section 6 .

The Neimark-Sacker curve also starts on that of Hopf points at $\sigma=0.028$ and $R a=$ 2910.77 at a double-Hopf bifurcation, as can be seen in Figure 2(b) and, in more detail, in Figure 2(c). The linear stability analysis of this point provided the initial conditions needed to follow the second curve of Hopf bifurcations giving rise to a second family of periodic orbits. By following them along paths of a fixed value of $\sigma$ or $R a$, we find initial conditions for the new saddle-node and Neimark-Sacker curves on this second family, also represented in the figures. The diagram obtained, close to the double-Hopf point, is that of the subregion II of the simple case of this codimension-two bifurcation as described in [21]. The upper limit of the new Neimark-Sacker curve is a strong 1:1 resonance point, located at $\sigma=0.617$, $R a=2174.91$, as will be seen later on a plot of the rotation number, $\theta / 2 \pi$, where $\theta$ is the phase of the crossing multiplier at the bifurcation.

A second movie (98101_02.gif [local/web 10.4MB]) shows the dynamics of the flow for the periodic orbit at $\sigma=0.5078$ and $R a=2182.20$ on the second Neimark-Sacker locus. It is a fixed cycle with respect to the transformation $R_{x} R_{y}$ and is a symmetric cycle with respect to $R_{x}$. The main difference from the first family is that there are now three vortices in the box instead of four.

The second curve of saddle-node points touches the second locus of Hopf bifurcations at $\sigma=0.012$ and $R a=3705.51$ at another Bautin point. The Hopf bifurcations are supercritical for $\sigma<0.012$ and subcritical for $\sigma>0.012$. With this information and that on the stability of the periodic orbits along the bifurcation loci presented in Table 1, it can be seen that there are stable periodic orbits of the second family, located above (larger $R a$ ) the second curve of saddle-nodes. This locus of saddle-node points intersects the second Neimark-Sacker curve at $\sigma=0.144$ at another fold-Hopf codimension-two point. At $\sigma=2.15$ the arclength steps taken by the continuation code were too small to continue the curve. We checked that there were no new multipliers approaching the unit circle on the saddle-node locus nor other nearby bifurcations on several sections of the second set of periodic orbits around this value of $\sigma$, computed by continuations in $R a$. This seems to indicate that multiple shooting is needed beyond this point due to the growing instability of the periodic orbits.

Figure 2(d) shows the period of the periodic orbits at their onset on the Hopf curves and along the curves of bifurcations. Since the periods along the former are different at the double-Hopf bifurcation, the curves passing through this point split into two groups in this representation. Globally, the period grows when $\sigma$ decreases to zero making the computations more expensive.

Table 1 summarizes the results on the stability of the periodic orbits along the bifurcation loci of Figure 2(b). The first column indicates the type of curve. The 1 and 2 refer to the first and the second family of limit cycles, respectively. The limits of the segments of the curves, 
Table 1

Stability of the periodic orbits on the bifurcation curves.

\begin{tabular}{cllcc} 
Bif. curve & Starting $\sigma$ & Ending $\sigma$ & Mult. on $S^{1}$ & Mult. out of $S^{1}$ \\
\hline SN1 & 0.122 & 1.699 & $1 \mathrm{r}$ & 0 \\
SN1 & 1.699 & 3.000 & $1 \mathrm{r}$ & $2 \mathrm{cc}$ \\
\hline NS1 & 0.028 & 1.699 & $2 \mathrm{cc}$ & 0 \\
NS1 & 1.699 & 3.000 & $2 \mathrm{cc}$ & $1 \mathrm{r}$ \\
\hline PF1 & 0.010 & 3.000 & $1 \mathrm{r}$ & $2 \mathrm{cc} / 2 \mathrm{r}$ \\
\hline SN2 & 0.012 & 0.144 & $1 \mathrm{r}$ & 0 \\
SN2 & 0.144 & 0.411 & $1 \mathrm{r}$ & $2 \mathrm{cc}$ \\
SN2 & 0.411 & 1.090 & $1 \mathrm{r}$ & $1 \mathrm{r}+2 \mathrm{cc} / 2 \mathrm{r}$ \\
SN2 & 1.090 & 2.150 & $1 \mathrm{r}$ & $2 \mathrm{r}$ \\
\hline NS2 & 0.028 & 0.144 & $2 \mathrm{cc}$ & $1 \mathrm{r}$ \\
NS2 & 0.144 & 0.190 & $2 \mathrm{cc}$ & 0 \\
NS2 & 0.190 & 0.617 & $2 \mathrm{cc}$ & $1 \mathrm{r}$ \\
\hline
\end{tabular}

separated by codimension-two points, are given by the Prandtl numbers in the second and third columns. The number of multipliers on and out of the unit circle are given in the last two columns. The symbols $1 \mathrm{r}, 2 \mathrm{r}$, and $2 \mathrm{cc}$ mean one real, two real, and two complex conjugate multipliers, respectively. The symbol $2 \mathrm{cc} / 2 \mathrm{r}$ indicates that two complex conjugate multipliers collide at the real axis becoming real, without crossing the unit circle.

Figure 3 shows the classical projection of the bifurcation curves on the parameter space $\sigma-R a$, with details of the three codimension-two points on the main family of periodic orbits. Specifically, Figures 3(b)-(d) display the details close to the Bautin, fold-Hopf of periodic orbits, and double-Hopf bifurcation points of the conduction state.

5.2. Computation of the boundaries of the 1/8-resonance region. With the tools described in section 3 it is also possible to follow the boundaries of resonance regions, since they are loci of saddle-node bifurcations. Figure 4 shows the continuation of the periodic orbits inside the resonance region of rotation number $1 / 8$, mentioned earlier in the description of Figure 1 for $\sigma=0.6$. A section of constant $R a$ in $2116.18<R a<2116.20$ gives 32 intersections, since due to the remaining symmetries there are two stable and two unstable periodic orbits on the invariant tori [40].

Two points on the boundaries of the interval were taken as initial conditions to follow the resonance region in the parameter $\sigma$. The results of the continuation are shown in Figure 5 . The saddle-node (in red) and the previously described Neimark-Sacker (in blue) curves can be seen in plot (a), projected onto the parameter space. The width of the Arnold's tongue is so tiny that a blow-up has been added to distinguish the two boundaries. Another possibility is to plot the norm $\|U\|$ versus $\sigma$. This representation highlights the presence of turning points on the upper curve, which do not completely develop in the lower (see Figure 5(b)). The period on the boundaries is shown in Figure 5(c). It increases rapidly when $\sigma$ decreases. This fact, together with the factor eight due to the resonance, makes the computation of these curves the most expensive of the calculations presented (see Figure 2(d) to compare the periods). Figure 5(d) displays the rotation number along the two curves of Neimark-Sacker bifurcations and the line $\rho=1 / 8$. On the second curve, it ends at $\rho=0$ and, as mentioned earlier, this point corresponds to a strong 1:1 resonance. 

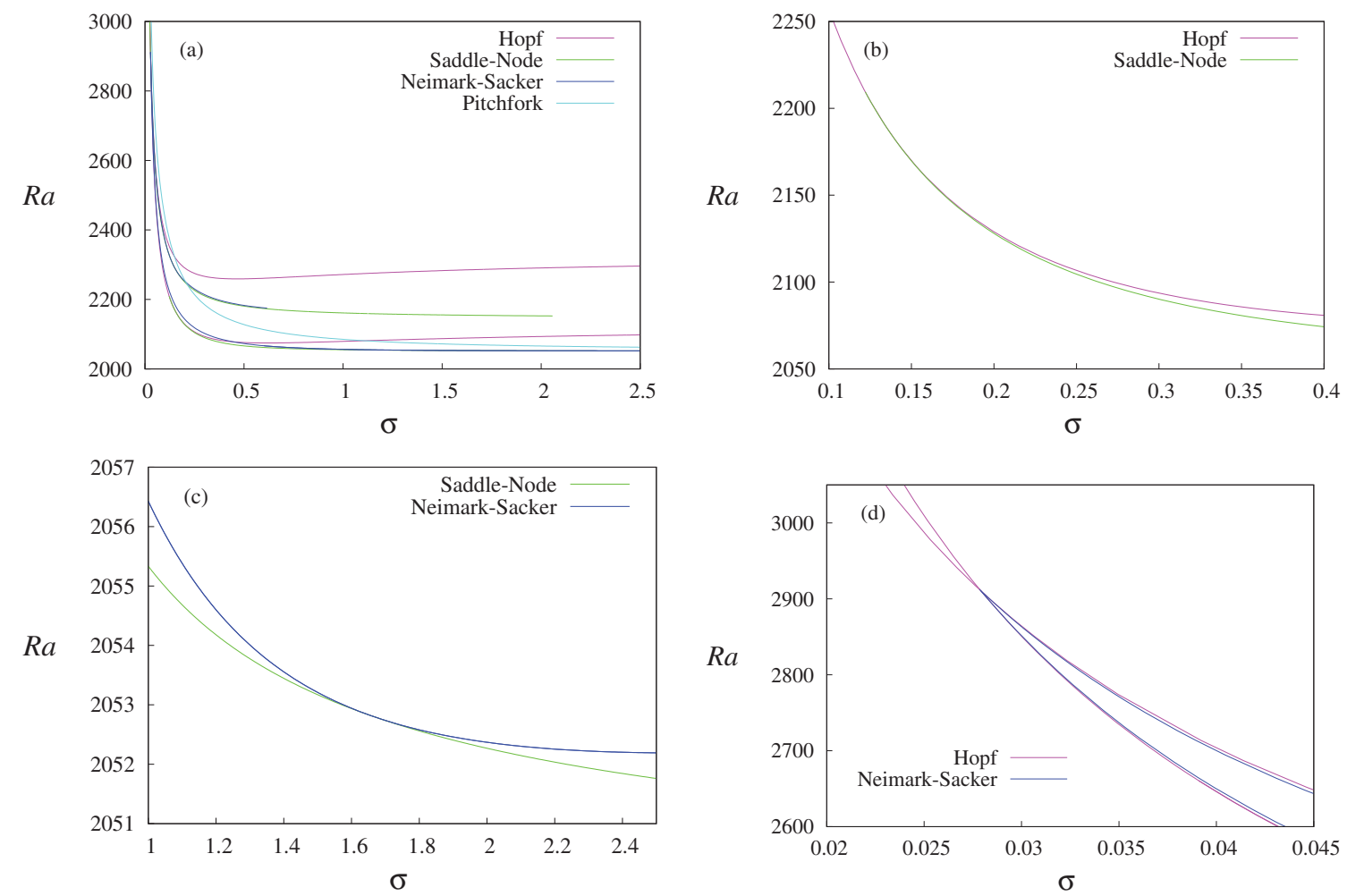

Figure 3. (a) Curves of bifurcations on the parameter space and details of three codimension-two points found on the main family of periodic orbits: (b) Bautin, (c) fold-Hopf of periodic orbits, and (d) double-Hopf bifurcations.

From the inspection of the characteristics of the periodic orbits (norms and period, for instance) of Figure 5(a) we have checked that the resonance region does not start on the upper Neimark-Sacker curve as might seem at first sight. In fact, we have found other Neimark-Sacker bifurcations at low $\sigma$, which could be the origin. It is known that in thermal convection problems with small $\sigma$, many bifurcations can accumulate close to the onset of convection producing very early chaotic behaviors.

Figure 6 shows several periodic orbits along one of the boundaries of the resonance region, projected onto the plane $\left(\Theta_{52,8}, \eta_{58,8}\right)$. The rectangle represented is $\left(\Theta_{52,8}, \eta_{58,8}\right) \in$ $[-0.04,0.04] \times[-0.25,0.15]$. The growth of their transverse diameter when $\sigma$ increases is obvious. In the lower right plot, all the intersections of the orbit with the hyperplane used as phase condition, $\psi_{38,9}=0$, have been included to make clear that there are 16 . Red squares and green circles indicate the two different crossing directions.

There is a single +1 multiplier on the unit circle along the two saddle-node curves, with the rest being stable, except between the two turning points, where there is a real positive multiplier out of the unit circle. Only below $\sigma \approx 0.128$ is there an unstable complex conjugate pair of multipliers. 


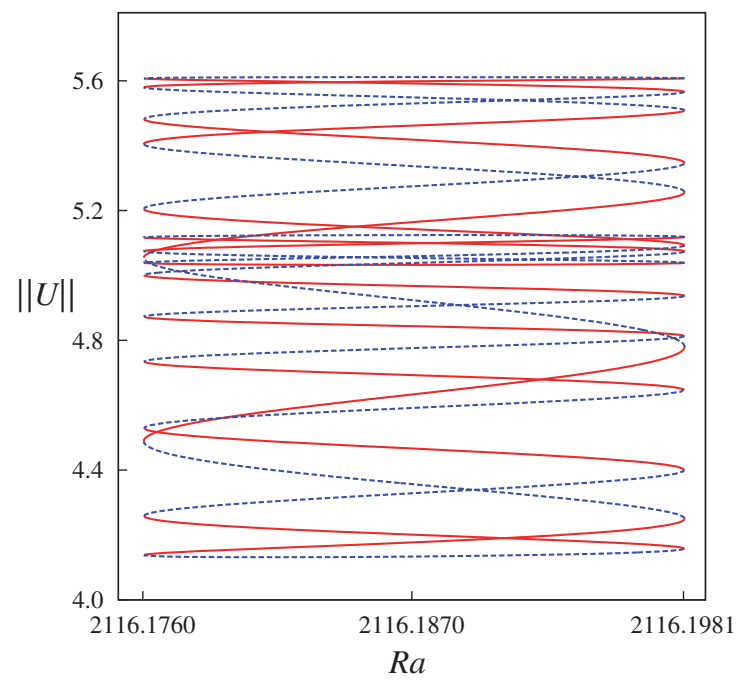

Figure 4. Continuation of the periodic orbits inside the $1 / 8$-resonance region for $\sigma=0.6$. Solid red and dashed blue lines correspond to stable and unstable periodic orbits, respectively.
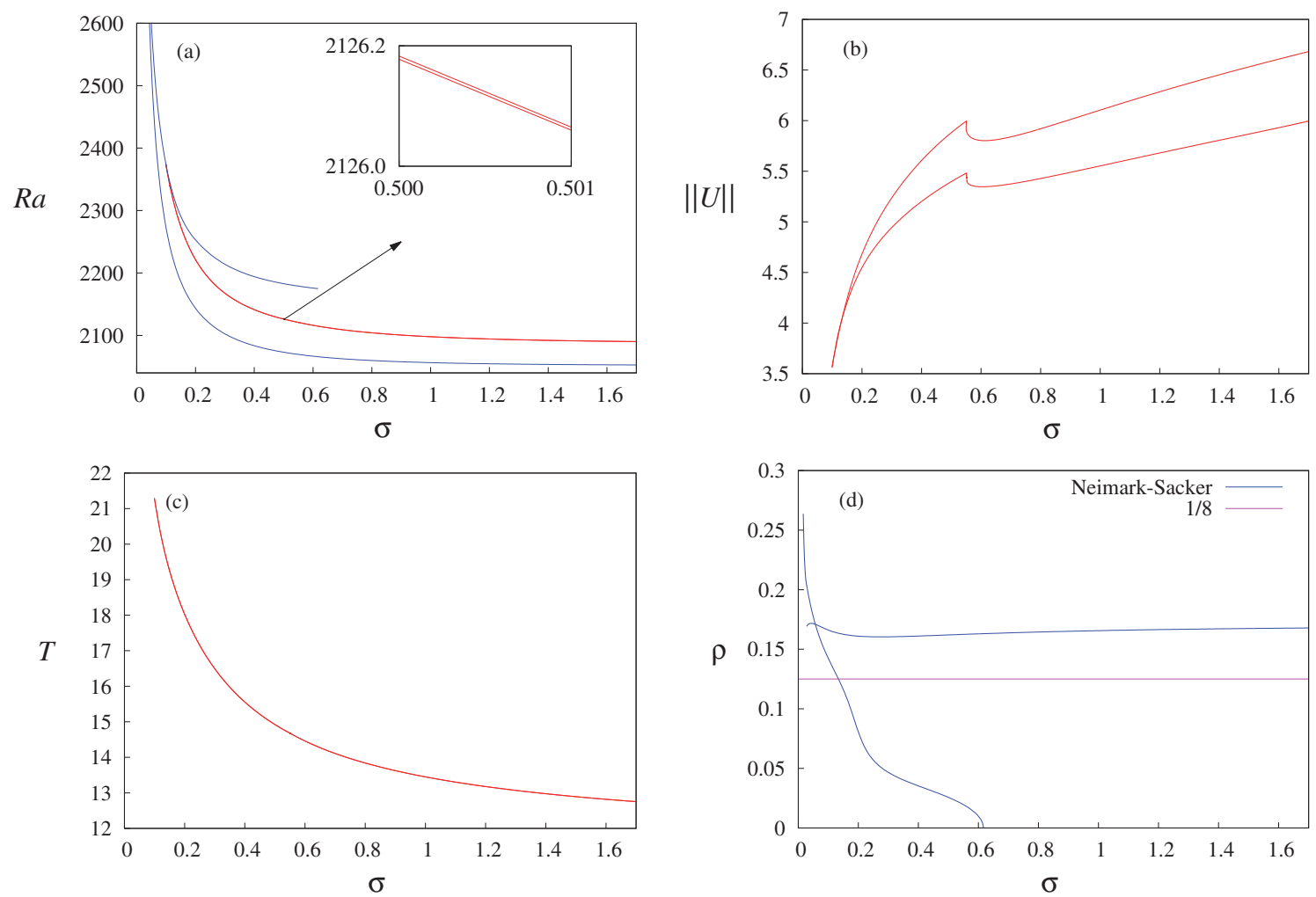

Figure 5. (a), (b) boundaries of the $1 / 8$ Arnold's tongue, (c) period, and (d) rotation number $\theta / 2 \pi$ along the Neimark-Sacker curves. 

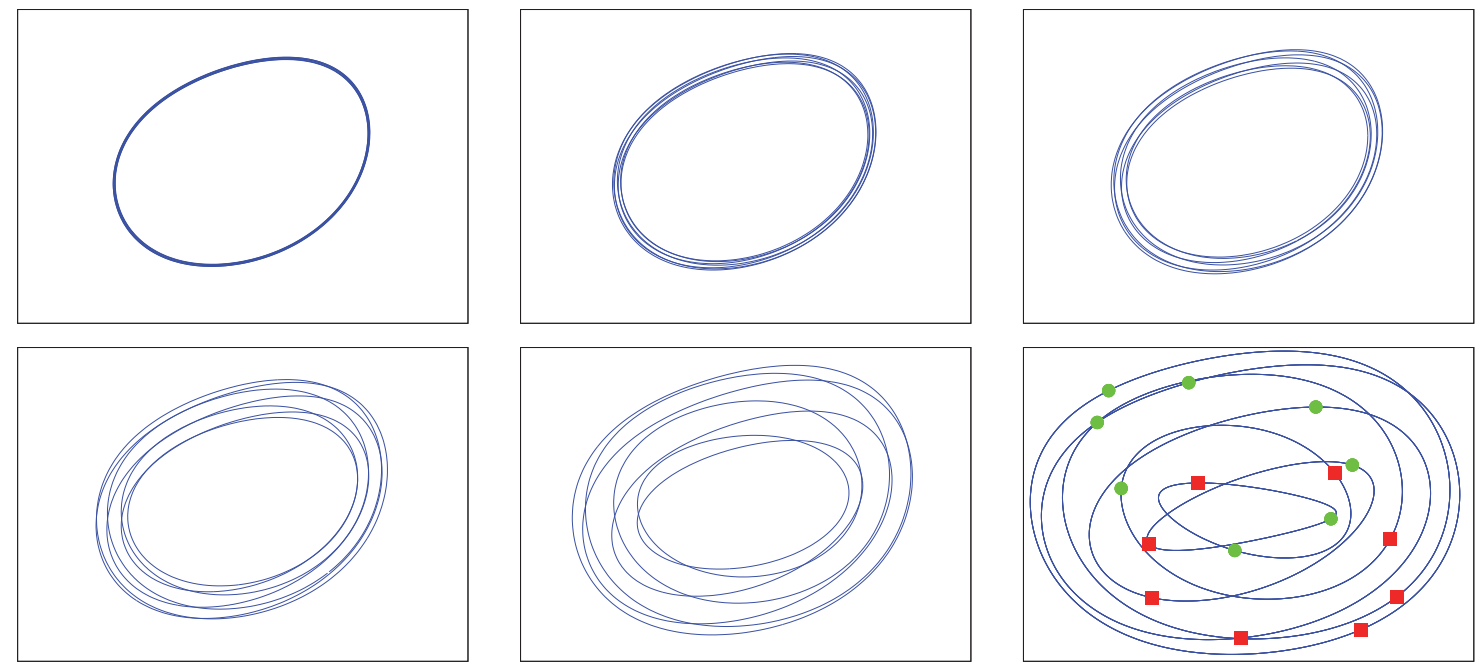

Figure 6. Projections of periodic orbits onto the variables $\left(\Theta_{52,8}, \eta_{58,8}\right)$ taken along the upper curve of Figure 5(b). The values of the parameters $(\sigma, R a)$ are $(0.0991,2374.56),(0.09965,2373.02),(0.1010,2369.28)$ (top, left to right), and $(0.1070,2353.63),(0.13175,2302.51),(0.2148,2210.32)$ (bottom, left to right).

6. Performance of the algorithms. Figure 7 shows two examples of the performance of the methods previously described for the test problem. Figures $7(\mathrm{a})$ and (b) show the relative distance between Newton's iterates after each iteration, $\left\|X^{k}-X^{k-1}\right\| /\left\|X^{k}\right\|, k=1, \ldots, 5$, (see caption for color codes) versus the point index for the curve of pitchfork bifurcations and for the second locus of Neimark-Sacker points described above (that ending in a 1:1 resonance at $\sigma=0.617)$. In the first case the cycles are always unstable and the extended system includes the deflation term and the symmetry-breaking parameter. The second corresponds to the augmented system of largest size, so one should expect an increased number of GMRES iterations. The horizontal line at $\varepsilon=10^{-8}$ represents the tolerance used to stop the iteration in these cases. When only $k$ iterations are needed, i.e., when the curve of the $k$ th iterate is below $\varepsilon$, the relative distance for the higher iterations is set to $10^{-16}$ to have continuous curves.

The curve of pitchfork bifurcations was computed in two parts, both starting at $\sigma=0.6$ and going to higher and lower values of this parameter. They are identified by the positive and negative indices. At the starting point the initial error was relatively large and the initial arclength step small. This is why there is a peak in the curves of relative distances and a small plateau of $\sigma$ at index $=0$ in Figure 7(a). As $\sigma$ goes to zero the corresponding Rayleigh number grows quickly (see Figure 3(a)), the initial error of the prediction also increases, and four iterations were often required. The solutions for $\sigma>4$ are close to the large $\sigma$ limit, and many of them were found in just two Newton's iterations.

The curve of Neimark-Sacker bifurcations was started close to the double-Hopf point at $\sigma=0.028$. Figure 7(b) shows that the highest number of Newton's iterations (five) was only needed for the first two points to start the branch and at the end when the singular resonance point is reached. There the continuation code shortens the arclength steps, as can be seen in the change of slope of the curve of $\sigma$ above $\sigma=0.6$. 

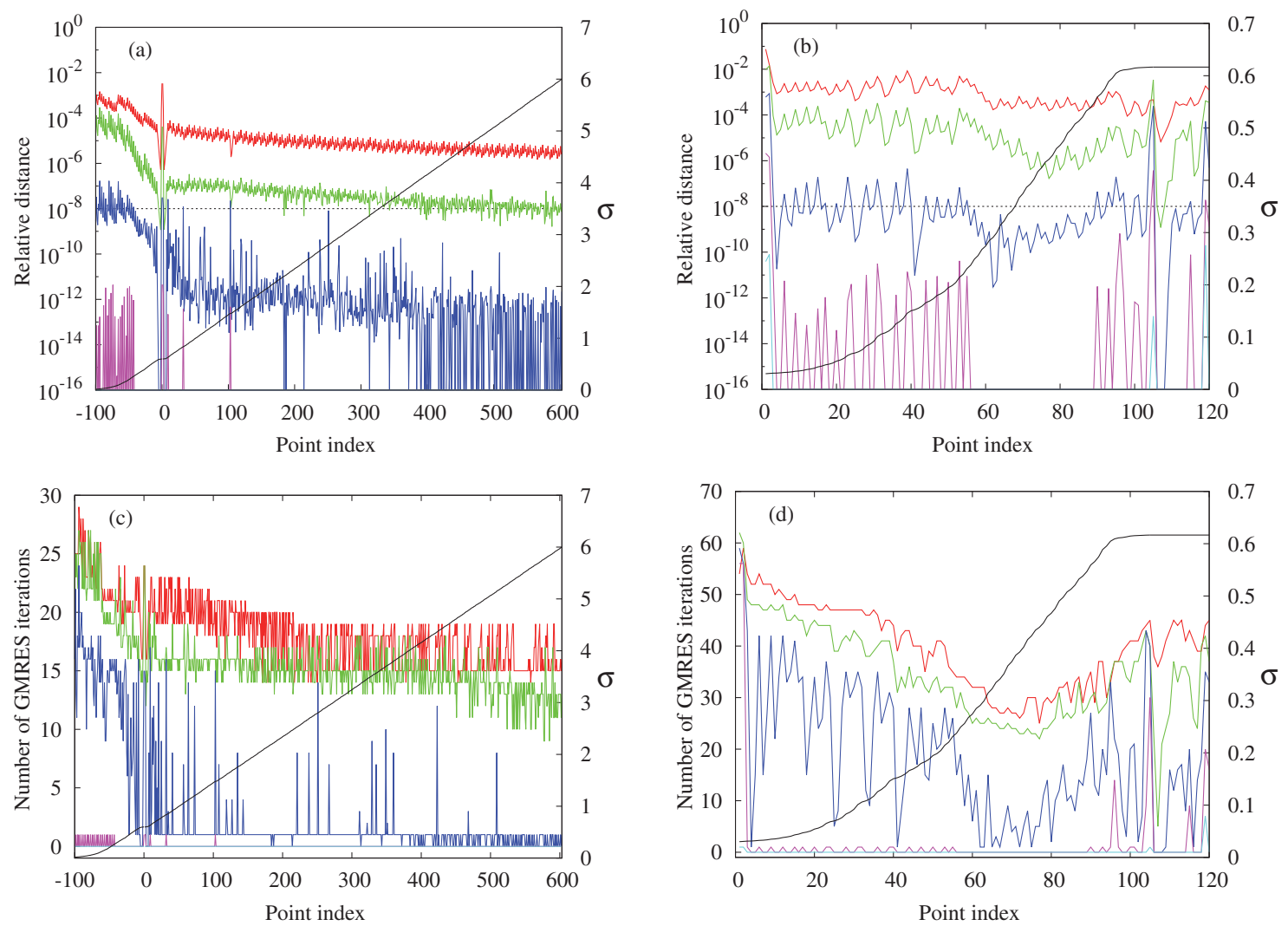

Figure 7. (a), (b) Relative distance between Newton iterates for the pitchfork and one of the NeimarkSacker curves, respectively, versus the index of the points along the curves. (c), (d) Corresponding number of GMRES iterations. Red, green, blue, magenta, and cyan correspond to the first, second, third, fourth, and fifth Newton's iteration, respectively. The horizontal line in black in (a) and (b) indicates the tolerance level in the computations. The curve in black in all plots represents the value of the Prandtl number $\sigma$ versus the index.

The quadratic convergence of Newton's method can be observed in Figures 7(a) and (b) except, as can be expected, close to bifurcation points where the Jacobian becomes near singular. The fourth or fifth iteration, when present, could not reach lower values because of the presence of time integration errors. Although being of high order (six in all calculations), errors are introduced in the evaluation of the explicit terms by matrix-matrix products and in the solution of the implicit terms.

Figures 7(c) and (d) show the number of GMRES(M) iterations needed to complete each Newton's step. An absolute criterion of convergence was used. The Euclidean norm of the residual of the linear systems was reduced to $\alpha \varepsilon$ with $\alpha=10^{-2}$ or $10^{-1}$, depending on the solutions computed, with $\varepsilon$ being the tolerance for Newton's method. The only requirement was keeping the quadratic convergence of the outer Newton's iterations. If the dimension of the Krylov subspace, M, is large enough, GMRES(M) finishes without restarting. The maximal dimension used was $M=70$ for the case of Neimark-Sacker points. In Figure 7(c) the maximal number of iterations required was always less than 30 , but it was less than 20 for most of the 
points, and the dimension of the system being solved was $m=2 n+4=6148$. In Figure $7(\mathrm{~d})$ the number of iterations was always less than 70 for a system of size $m=3 n+4=9220$.

The reason for the fast convergence of GMRES was explained in detail in $[38,36]$ for the computation of periodic orbits and in $[40,37]$ for that of invariant tori for the serial and parallel implementations. In particular, in [38] an upper bound was presented for the number of iterations needed by GMRES in the computation of periodic orbits. It was based on the knowledge of the distribution of the multipliers of the cycles. In [37] we used the results in [3] to explain the reason for the independence of the number of GMRES iterations with the number of points used to approximate an arc of an invariant curve. The main idea is the same in all of these cases and for the methods presented. All of these algorithms solve high-dimensional fixed-point problems of the form $X-G(X)=0$, where $G$ is a map obtained by integrating a system of truncated parabolic PDEs, with a few more additional equations (phase conditions, normalizing conditions for the eigenvectors, pseudoarclength conditions, etc.). For instance, in the case of the saddle-node points, the map is (see (11) and (13))

$$
(x, u) \rightarrow G(x, u)=\left(\varphi(T, x, p), D_{x} \varphi(T, x, p) u-w\langle w, u\rangle /\langle w, w\rangle\right),
$$

with phase and normalizing conditions given by (12) and (14), and $X=(x, u)$. Since the flow of the evolutionary equations defining $G$ strongly contracts volumes, $D_{X} G$ has most of its spectrum tightly clustered around zero. The matrix of the linear systems solved at each Newton's iteration is the Jacobian $I-D_{X} G(X)$, bordered, for the extended systems presented here, with at most three more rows and columns. The matrix $I-D_{X} G(X)$ can be seen as the identity, $I$, plus a low rank matrix $K$, the action of $-D_{X} G(X)$ restricted to the subspace corresponding to the eigenvalues of $D_{X} G(X)$ of modulus greater than a small $\varepsilon^{\prime}$, plus a perturbation matrix of small norm $E$, the action of $-D_{X} G(X)$ restricted to the subspace corresponding to the eigenvalues of $D_{X} G(X)$ of modulus less than $\varepsilon^{\prime}$. It was proved (see $[32,3]$ among others) that for systems with clusters of eigenvalues, GMRES converges quickly. Moreover, it was shown in [8] that for systems of the form $I+K+E$ like those just described, the addition of a row and a column only adds a few units to the number of iterations of GMRES needed to solve a system with the original matrix.

In our particular test problem, the similar structure of the original problem (40)-(42) and that of the variational equations in section 4.1, which differ only in the low order forcing terms, makes the spectrum of the maps defining the extended systems strongly clustered. The particular number of relevant eigenvalues, i.e., the rank of $K$, and therefore the number of iterations needed by GMRES and the time necessary to compute each bifurcation curve, depend on many factors. Two of them are the number of multipliers of the critical cycle of modulus larger than a small $\varepsilon^{\prime}$, and the number of variational equations involved in the computation of $G$. When the periodic orbits are very unstable, more iterations might be required by the linear solver to converge, and shorter arclength steps are usually taken. This was the case, for instance, for the orbits on the pitchfork curve, which have two unstable multipliers. In any case, the computational cost is affordable. The CPU time needed to complete each of the bifurcation curves, using Intel I7 processors with frequencies in the range of 2.67 to $3.2 \mathrm{GHz}$ and tolerances for the distance between Newton's iterates between $10^{-10}$ and $10^{-8}$, was from about two days for the tracking of Hopf points to a week and a half for the more expensive Neimark-Sacker case. The continuation of the boundaries of resonance 
regions was heavier. Since the period is multiplied by eight, the CPU time was also multiplied by this factor.

For simplicity, no parallelism was used in our calculations. There are several tasks that can be done concurrently. Systems (24)-(27) and (24), (28) can be solved in parallel. The different products in the action by the Jacobian of the extended systems can be computed separately. An inspection of the variational equations of section 4.1, for the problem presented here, reveals that the computation of the forcing terms of each equation can also be done in parallel, and that the linear systems, which must be solved at each time step, decouple into smaller ones because of the semi-implicit time stepper used. They can also be solved simultaneously.

7. Final remarks. The methods presented here are very efficient. The number of equations to integrate is limited to a minimum, Newton's quadratic convergence is preserved, and the linear solver converges very quickly. Moreover, they fit into the simple framework of NewtonKrylov methods. The main requirement is to have a time integration method that properly solves the systems of equations defining the function and the action by its Jacobian. Three main causes of breakdown of the algorithms must be taken into account, the presence of codimension-two bifurcation points that cannot be overshot because the curve followed does not continue at the other side, the increase of the instability of the periodic orbits, and the loss of transversality of the phase condition. The first is common to any continuation problem, the second can be solved by multiple shooting, and the third can be solved by changing adaptively the phase condition.

The extended systems presented in section 3 have been used to follow geometrical loci of codimension-one bifurcations, either of periodic orbits or fixed points. In the latter case the present formulation may be the best option if, for instance, there is no good preconditioner for the classical methods of tracking bifurcations of fixed points, or if a previous code for time integration or for the computation of periodic orbits, which needs only slight adjustments, is available. In our case, the computation of the curves of Hopf bifurcations of the conduction state of Figure 2 required the shortest CPU time.

Several codimension-two points were found during the computation of the bifurcation loci of periodic orbits. Some types are easy to detect because the conditions defining them depend only on the multipliers crossing or moving on the unit circle. This is the case of the two foldHopf points of cycles, and the 1:1 resonance for which the phase of the critical multiplier being followed vanishes, shown in Figure 2(b). The ending of a saddle-node locus at a Bautin point is also easy to detect because the diameter of the periodic orbits collapses to zero when the curve of Hopf bifurcations is reached. A lower bound for this diameter can be easily computed during the time evolution of a cycle by calculating the distance of each point of the numerical integration to the initial condition. A cusp point is also easy to see by looking at the projection onto the two-parameter space of the saddle-node curve being followed. In the case of lowdimensional systems of ODEs, other codimension-two bifurcations that cannot be classified by just looking at the spectrum and that depend on degeneracy conditions are detected by using test functions. They need the computation of the critical eigenvectors of an adjoint operator $[21,16]$. In the case of periodic orbits it is $\left(D_{x} \varphi(T, x, p)\right)^{*}$. This is a difficult task in PDEs when the base cycle is only known numerically. Therefore it cannot be suggested as a 
general methodology. The work [41] is the only publication we know of in which this has been done. Moreover, the extended systems for the codimension-one bifurcations are regular at the codimension-two points corresponding to the vanishing of normal form coefficients (see [21]). Consequently, they are overshot when tracing codimension-one loci, and the computation of the leading eigenvalues of the Jacobian of the augmented systems is, in general, not helpful to signal these codimension-two singularities. If a rectangular region of parameters is to be explored, the combination of continuations of cycles for two families of sections at constant values of one of the two parameters, followed by the study of their stability together with the continuation of the codimension-one points detected, can be helpful to complete the bifurcation diagram of the system.

As mentioned earlier, the continuation of codimension-one bifurcations of periodic orbits can also be formulated in terms of the Poincaré map, with the advantage of eliminating the need to deflate the extra +1 eigenvalue, but with the disadvantages of introducing projections to compute the derivatives and of needing to parameterize the map (see [38]).

It is important to realize that, for systems depending on three parameters, the extended systems presented can be enlarged with extra equations (already written in some of the systems) to continue the loci of codimension-two points when they are defined exclusively by the spectrum of the solution at the bifurcation point, eventually including generalized eigenvectors. This is the case, for instance, of the double-Hopf of the conduction state and the fold-Hopf bifurcation of periodic orbits of Figure 2. Further investigation is required to understand how to apply matrix-free Newton-Krylov techniques for the continuation of bifurcations of fixed points or periodic orbits of large-scale systems in order to include degeneracy conditions as happens, for instance, with Bautin or Chenciner points.

\section{REFERENCES}

[1] R. Barrett, M. Berry, T. F. Chan, J. Demmel, J. Donato, J. Dongarra, V. Eijkhout, R. Pozo, C. Romine, And H. Van der Vorst, Templates for the Solution of Linear Systems: Building Blocks for Iterative Methods, SIAM, Philadelphia, 1994.

[2] K. Böhmer, Z. Mei, A. Schwarzer, And R. Sebastian, Path-following of large bifurcation problems with iterative methods, in Numerical Methods for Bifurcation Problems and Large-Scale Dynamical Systems, E. Doedel and L. S. Tuckerman, eds., IMA Vol. Math. Appl. 119, Springer, Berlin, 2000, pp. $35-65$.

[3] S. L. Campbell, I. C. F. Ipsen, C. T. Kelley, and C. D. Meyer, GMRES and the minimal polynomial, BIT, 36 (1996), pp. 664-675.

[4] K. A. Cliffe, Numerical calculations of the primary-flow exchange process in the Taylor problem, J. Fluid Mech., 197 (1988), pp. 57-79.

[5] K. A. Cliffe, A. Spence, And S. J. Taverner, The numerical analysis of bifurcation problems with applications to fluid mechanics, Acta Numer., 9 (2000), pp. 39-131.

[6] S. R. De Groot and P. Mazur, Non-Equilibrium Thermodynamics, Dover Publications, Amsterdam, 1962.

[7] A. Dhooge, W. J. F. Govaerts, And Y. A. Kuznetsov, MATCONT: A MATLAB package for numerical bifurcation analysis of ODEs, ACM Trans. Math. Soft., 29 (2003), pp. 141-164.

[8] K. I. Dickson, C. T. Kelley, I. C. F. Ipsen, And I. G. Kevrekidis, Condition estimates for pseudoarclength continuation, SIAM J. Numer. Anal., 45 (2007), pp. 263-276.

[9] H. A. Dijkstra, F. W. Wubs, A. K. Cliffe, E. Doedel, I. F. Dragomirescu, B. Eckhardt, A. Gelfgat, A. Hazel, V. Lucarini, A. Salinger, J. Sánchez, H. Schuttelaars, L. TuckERMAN, AND U. ThiELE, Numerical bifurcation methods and their application to fluid dynamics: Analysis beyond simulation, Commun. Comput. Phys., 15 (2014), pp. 1-45. 
[10] E. Doedel, AUTO: Software for Continuation and Bifurcation Problems in Ordinary Differential Equations, Tech. report, Applied Mathematics, California Institute of Technology, Pasadena, CA, 1986.

[11] E. J. Doedel, W. Govaerts, And Yu. A. Kuznetsov, Computation of periodic solution bifurcations in ODEs using bordered systems, SIAM J. Numer. Anal., 41 (2003), pp. 401-435.

[12] W. S. Edwards, L. S. Tuckerman, R. A. Friesner, And D. C. Sorensen, Krylov methods for the incompressible Navier-Stokes equations, J. Comput. Phys., 110 (1994), pp. 82-102.

[13] K. Engelborghs, K. Lust, And D. Roose, Direct computation of period doubling bifurcation points of large-scale systems of ODEs using a Newton-Picard method, IMA J. Numer. Anal., 19 (1999), pp. 525-547.

[14] V. Frayssé, L. Giraud, S. Gratton, and J. Langou, Algorithm 842: A set of GMRES routines for real and complex arithmetics on high performance computers, ACM Trans. Math. Softw., 31 (2005), pp. 228-238.

[15] B. García-Archilla, J. Sánchez, and C. Simó, Krylov methods and test functions for detecting bifurcations in one parameter-dependent partial differential equations, BIT, 46 (2006), pp. 731-757.

[16] W. J. F. Govaents, Numerical Methods for Bifurcations of Dynamical Equilibria, SIAM, Philadelphia, 2000.

[17] K. R. Green and L. Van Veen, Open-source tools for dynamical analysis of Liley's mean-field cortex model, J. Comput. Sci., 5 (2014), pp. 507-516.

[18] A. Griewank And G. Reddien, The calculation of Hopf points by a direct method, IMA J. Numer. Anal., 3 (1983), pp. 295-303.

[19] G. Kawahara, M. Uhlmann, and L. VAn Veen, The significance of simple invariant solutions in turbulent flows, Ann. Rev. Fluid Mech., 44 (2012), pp. 203-225.

[20] H. B. KelleR, Numerical solution of bifurcation and nonlinear eigenvalue problems, in Applications of Bifurcation Theory, P. H. Rabinowitz, ed., Academic Press, New York, 1977, pp. 359-384.

[21] Y. A. Kuznetsov, Elements of Applied Bifurcation Theory, 2nd ed., Springer, Berlin, 1998.

[22] Y. A. Kuznetsov and V. V. Levitin, CONTENT: A Multiplatform Continuation Environment, Tech. report, CWI, Amsterdam, The Netherlands, 1996.

[23] Y. A. Kuznetsov, H. G. E. Meijer, and L. van Veen, The fold-flip bifurcation, Int. J. Bifur. Chaos, 14 (2004), pp. 2253-2282.

[24] J. M. Lopez, F. Marqués, And J. SÁnchez, Oscillatory modes in an enclosed swirling flow, J. Fluid Mech., 439 (2001), pp. 109-129.

[25] K. Lust, D. Roose, A. Spence, And A. R. Champneys, An adaptive Newton-Picard algorithm with subspace iteration for computing periodic solutions, SIAM J. Sci. Comput., 19 (1998), pp. 1188-1209.

[26] G. Moore And A. Spence, The calculation of turning points of nonlinear equations, SIAM J. Numer. Anal., 17 (1980), pp. 567-576.

[27] R. P. Pawlowski, J. N. Shadid, J. P. Simonis, and H. F. Walker, Globalization techniques for Newton-Krylov methods and applications to the fully coupled solution of the Navier-Stokes equations, SIAM Rev., 48 (2006), pp. 700-721.

[28] D. Puiguaner, J. Herrero, C. Simó, and F. Giralt, From steady solutions to chaotic flows in a Rayleigh-Bénard problem at moderate Rayleigh numbers, Phys. D, 240 (2011), pp. 920-934.

[29] D. Roose And V. HLAvaČEK, A direct method for the computation of Hopf bifurcation points, SIAM J. Appl. Math., 45 (1985), pp. 879-894.

[30] Y. SAAD, Numerical Methods for Large Eigenvalue Problems, Manchester University Press, Manchester, 1992. Updated and revised edition published as Classics Appl. Math. 66, SIAM, Philadelphia, 2011.

[31] Y. SAAD, Iterative methods for sparse linear systems, PWS, New York, 1996.

[32] Y. SAAd And M. H. Schultz, GMRES: A generalized minimal residual algorithm for solving nonsymmetric linear systems, SIAM J. Sci. Stat. Comput., 7 (1986), pp. 856-869.

[33] A. G. Salinger, N. M. Bou-Rabee, R. P. Pawlowsky, E. D. Wilkes, E. A. Burroughs, R. B. Lehoucq, And L. A. Romero, LOCA 1.1. Library of Continuation Algorithms: Theory and Implementation Manual, Sandia National Laboratories, Albuquerque, NM, 2002.

[34] A. G. Salinger, E. A. Burroughs, R. P. Pawlowski, E. T. Phipps, And L. A. Romero, Bifurcation tracking algorithms and software for large scale applications, Int. J. Bifur. Chaos, 15 (2005), pp. 10151032. 
[35] J. SÁnchez, F. Garcia, And M. Net, Computation of azimuthal waves and their stability in thermal convection in rotating spherical shells with application to the study of a double-Hopf bifurcation, Phys. Rev. E, 87 (2013), 033014.

[36] J. SÁnchez And M. Net, On the multiple shooting continuation of periodic orbits by Newton-Krylov methods, Int. J. Bifur. Chaos, 20 (2010), pp. 1-19.

[37] J. SÁnchez AND M. NET, A parallel algorithm for the computation of invariant tori in large-scale dissipative systems, Phys. D, 252 (2013), pp. 22-33.

[38] J. Sánchez, M. Net, B. García-Archilla, And C. Simó, Newton-Krylov continuation of periodic orbits for Navier-Stokes flows, J. Comput. Phys., 201 (2004), pp. 13-33.

[39] J. Sánchez, M. Net, B. García-Archilla, and C. Simó, Continuation of periodic orbits in largescale dissipative systems, in Proceedings of the Equadiff-2003 Conference, F. Dumortier, H. Broer, J. Mawhin, A. Vanderbauwhede, and S. Verduyn Lunel, eds., World Scientific, Singapore, 2005, pp. 625-630.

[40] J. SÁnchez, M. Net, And C. Simó, Computation of invariant tori by Newton-Krylov methods in largescale dissipative systems, Phys. D, 239 (2010), pp. 123-133.

[41] J. SÁnchez, M. Net, And J. M. VegA, Amplitude equations close to a triple- $(+1)$ bifurcation point of $D_{4}$-symmetric periodic orbits in $O(2)$-equivariant systems, Discrete Contin. Dyn. Syst. B, 6 (2006), pp. $1357-1380$.

[42] R. SEydel, Numerical computation of branch points in nonlinear equations, Numer. Math., 33 (1979), pp. 339-352.

[43] R. Seydel, From Equilibrium to Chaos: Practical Bifurcation and Stability Analysis, Elsevier, New York, 1988.

[44] C. Simó, On the analytical and numerical computation of invariant manifolds, in Modern Methods in Celestial Mechanics, D. Benest and C. Froeschlé, eds., Editions Frontières, Paris, 1990, pp. 285-329. Available online at http://www.maia.ub.es/dsg/2004/.

[45] L. S. Tuckerman And D. BARKLEy, Bifurcation analysis for timesteppers, in Numerical Methods for Bifurcation Problems and Large-Scale Dynamical Systems, E. Doedel and L. S. Tuckerman, eds., vol. 119 of IMA Volumes in Mathematics and its Applications, Springer-Verlag, 2000, pp. 453-466.

[46] T. L. van Noorden, S. M. Verduyn Lunel, and A. Bliek, The efficient computation of periodic states of cyclically operated chemical processes, IMA J. Appl. Math., 68 (2003), pp. 149-166.

[47] L. van Veen, G. Kawahara, and M. Atsushi, On matrix-free computation of $2 D$ unstable manifolds, SIAM J. Sci. Comput., 33 (2011), pp. 25-44.

[48] D. Viswanath, Recurrent motions within plane Couette turbulence, J. Fluid Mech., 580 (2007), pp. 339358.

[49] T. Watanabe, M. Ima, and Y. Y. Nishiura, Spontaneous formation of travelling localized structures and their asymptotic behaviour in binary fluid convection, J. Fluid Mech., 712 (2012), pp. 219-243.

[50] T. Watanabe and S. Yanase, Bifurcation study of three-dimensional solutions of the curved square-duct flow, J. Phys. Soc. Japan, 82 (2013), 074402.

[51] I. Waugh, S. Illingworth, AND M. Juniper, Matrix-free continuation of limit cycles for bifurcation analysis of large thermoacoustic systems, J. Comput. Phys., 240 (2013), pp. 225-247.

[52] I. C. Waugh, K. Kashinath, And M. P. Juniper, Matrix-free continuation of limit cycles and their bifurcations for a ducted premixed flame, J. Fluid Mech., 759 (2014), pp. 1-27.

[53] B. Werner And A. Spence, The computation of symmetry-breaking bifurcation points, SIAM J. Numer. Anal., 21 (1984), pp. 388-399. 